\title{
Superconductivity: Chronology of Events and Hallmrk Developments
}

\author{
Kul Prasad Dahal \\ Tribhuan University, Prithvi Narayan Campus, Pokhara \\ Email:dahalkp@yahoo.com
}

\section{Introduction}

When metals are cooled to a lower temperature some of them exhibit an abrupt drop in resistivity and enter a state in which there is no resistance to the flow of the electric current beyond certain temperature, known as critical temperature $(\mathrm{Tc})$. Below critical temperature, a phase transition occurs. This new phase was first discovered in mercury by $\mathrm{H}$ Kammerlingh Onnes at the University of Leiden in the spring of 1911. It was just three years after the first liquefaction of helium. K Onnes noted that, upon cooling below 4.2 Kelvin "Mercury has passed into a new state, which on account of its extraordinary properties may be called a superconductive state". In superconducting state the electrical resistivity suddenly drops to an immeasurable small value $(\sim 10$ $\left.{ }^{-25} \Omega \mathrm{m}\right)$. The temperature at which the transition of the state from normal to the superconducting state occurs is not same for all superconducting materials. The critical temperature varies from one to another superconductor but its range lies between less than $1 \mathrm{~K}$ and approximately $20 \mathrm{~K}$ for metals and metallic alloys. The transition from the normal to the superconducting phase is often sharp and occurs within $10^{-2} \mathrm{~K}$ to $10^{-4} \mathrm{~K}$. The sharpness of superconducting state transition depends on the state and purity of the sample. The superconducting state exhibits both electromagnetic (zero resistance, persistence current, critical field, Meissner effect) as well as thermal properties (heat capacity, entropy, thermal conductivity, energy gap).

Superconductivity is a macroscopic quantum phenomenon. There was no possibility of explaining the phenomenon before the advent of quantum mechanics in the mid twenties. Quantum theories in which matter has both particle and wave aspects discovered by Heisenberg (1924) and Schrodinger (1925) was soon applied to transport of electricity by electrons in metal but superconductivity remained a mystery. In 1950 it was shown that superconductivity involves an interaction between the conduction electrons and vibrational motion of the ions in the metal. In 1957 L Cooper, R Schrieffer and J Bardeen develop a satisfactory theory of superconductivity knownas BCStheory, aftertheinitialsofthediscoverer.

Superconductor may be an element, inter-metallic alloy and semiconductor, compound of organic or inorganic substance that will conduct electricity without resistance below a certain temperature. All known superconductors are solids, none are gases or liquids, all require extreme cold to enter superconductive state.

\section{The signature of superconducting state i) The Meissner effect and zero resistance}

This is a classic hallmark of superconductivity, the Meissner effect exhibiting diamagnetic properties to the total exclusion of all magnetic fields. The Meissner effect was discovered by Meissner and Ochsenfeld in 1933. They found that the magnetic field is pushed out of the bulk of superconductor. This means, the magnetic induction (B) inside the superconductor is always zero. A superconductor is a perfect diamante having magnetic susceptibility negative and less than unity (since, $\chi=-1 / 4 \pi$ ). On the other hand electric resistivity of the superconducting material drops to zero, which accounts for a constant magnetic field inside the superconducting material. But the disappearance of resistivity and the expulsion of magnetic flux are two independent phenomena but essential properties of the superconducting state. The superconductivity depends on former phenomena to maintain the later one. The Meissner effect is reversible and the temperature transition from the normal state to the superconducting state and vice versa depends on the strength of the applied magnetic field. The flux expulsion continues to all the temperatures $\mathrm{T}$ less than critical temperatures $\mathrm{T}_{\text {c. }}$ Above the critical temperature penetration of magnetic flux begins. When the temperature is 
raised from below $T_{c}$, the flux suddenly penetrates the specimen after it reaches $T_{c}$ and the material turns into normal state. A beautiful demonstration of flux expulsion can be seen when a magnet resting on the superconducting specimen, on cooling it below $\mathrm{T}_{c}$ the magnet floats in the air above the specimen without touching, so as to push the magnetic flux out of the magnet. The magnet comes back to rest on the specimen on increasing the temperature above $T_{c}$.

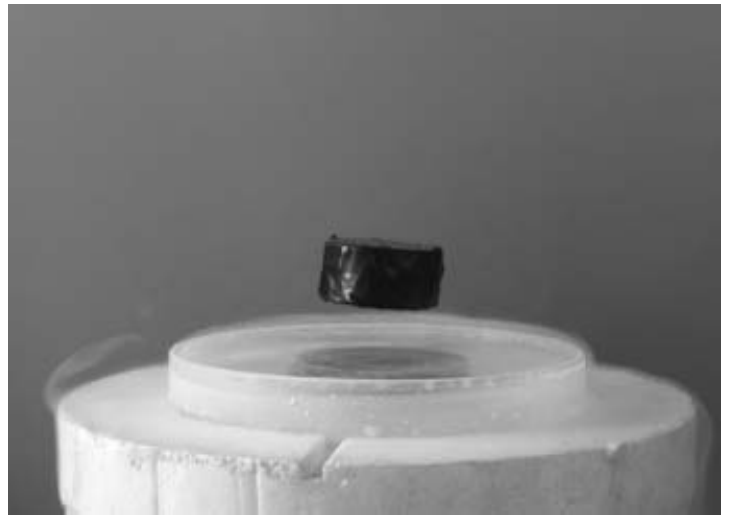

Fig.1: An illustration of Meissner effect, showing floating of a magnet in the air above the superconducting specimen, when it is at a temperature below $T_{c}$.

\section{ii) Persistent Current}

Work at Massachusetts Institute of Technology (MIT) showed, a dc current of several hundred amperes once induced in a superconducting ring continue to flow without attenuation for a whole year. Such type of current is called persistent current .The existence of persistent electrical current is the first traditional hallmark of Superconductivity. Persistent current can easily be observed in a superconducting ring. It is to be noted that zero resistivity and persistent currents are one and the some property of the superconductors.

The current $I(t)$ flowing in the ring at time $t$ is $-\mathrm{A}(\mathrm{dB} / \mathrm{dt})=\mathrm{RI}(\mathrm{t})+\mathrm{LdI}(\mathrm{t}) / \mathrm{dt}$, where $\mathrm{A}$ is the area of the ring, $\mathrm{R}$ is the resistance and $\mathrm{L}$ is the inductance of the ring. If there is no external applied magnetic field then,

$$
R(t)+L \frac{d(t)}{d t}=0
$$

Thas a solution,

$$
I(t)=I(0)-e^{-\cdot \frac{R \cdot t}{L}}
$$

In the superconducting ring $\mathrm{R}=\mathrm{O}$ then $\mathrm{I}(\mathrm{t})=\mathrm{I}(0)$. It gives the meaning of never attenuation of current with time. Persistent currents remain without change, within the limits of detection by the most sensitive modern instruments, for many years. The creep rates of current are so small that the super currents in $\mathrm{Nb}_{3} \mathrm{Sn}$ would die out after time more than $3 \times 10^{92}$ years, which is far more than the age of the earth. Therefore in practical sense the super currents can be considered as persistent current.

\section{iii) Thermal Conductivity}

There is an abrupt change in thermal conductivity at $\mathrm{T}_{\mathrm{c} .}$ In some superconductors the thermal conductivity increases as the temperature decreases below $T_{c}$, while in other superconductors thermal conductivity decreases with temperature below Tc. Experimentally it was observed that for type - I superconductors there is a marked drop in the thermal conductivity when superconductivity sets in, suggesting that the electronic contribution drops in, the superconducting electrons possibly playing no part in heat transfer.

\section{iv) Entropy}

As entropy is measure of the disorder of a system. In all superconductors, entropy decreases considerably upon cooling below $\mathrm{T}_{\mathrm{c}}$. Superconducting state is more ordered than the normal state i.e. fraction of electrons that is thermally excited in the normal state becomes ordered in the superconducting state. The change in entropy is small (in Aluminum $\sim 10^{-4} \mathrm{~K}_{\mathrm{B}}$, here $\mathrm{K}_{\mathrm{B}}$ is Boltzmann constant). The small entropy change must mean that only a small fraction of the conduction electrons participate in the transition to the superconducting state.

\section{v) Energy Gap}

The existence of the energy gap in superconductors has been confirmed by a number of experiments. Energy gaps in superconductors are of entirely different nature than the energy gap in insulators. In superconductors energy gap separates superconducting electron states below it from the normal electron states lying above it. Moreover this gap decreases continuously to zero, as the temperature is increased to the critical temperature 
$\left(\mathrm{T}_{\mathrm{c}}\right)$. The transition at zero fields is second order but the transition in the presence of an applied magnetic field is first order, since there are discontinuities in state properties as well as an associated latent heat. The thermodynamic energy difference between the normal and superconducting state is called condensation energy of the superconducting state. This is the energy required to break up a pair of electrons. According to BCS theory the energy gap is $3.5 \mathrm{KT}_{\mathrm{c}}$, where $\mathrm{K}$ is Boltzman constant.

\section{vi ) Specific Heat Capacity}

The temperature dependence of the specific heat in the superconducting state is very much different from that in the normal state. There occurs an abrupt increase in specific heat capacity at temperature $T_{c}$. The electronic specific heat above $\mathrm{T}_{\mathrm{c}}$ and below $\mathrm{T}_{\mathrm{c}}$ is of quiet different in nature. In the former case specific heat is linearly related with temperature $\left\{\mathrm{C}_{\mathrm{en}}=\gamma \mathrm{T}\right\}$, whereas in latter case it is related exponentially with the temperature $\left\{\mathrm{C}_{\mathrm{es}}=\exp \left(-\Delta / \mathrm{K}_{\mathrm{B}} \mathrm{T}\right), \Delta\right.$-energy gap $\}$.

\section{Theoretical Developments}

Theories of superconductivity developed along two lines. The phenomenological theories of London, Pippard, Ginzburg and Landau deal with the existence and order of magnitude of the penetration depth and coherence length and give a qualitative account of the electrodynamic properties. The microscopic theory of Frohlich introduced the concept of an interaction between superconducting electrons and phonons. The BCS theory accounts for the observed properties of superconductors. The result of the phenomenological theories can be derived from BCS theory, and superconductivity can also be understood in terms of the electronic and atomic structures. Josephson and Anderson discovered the importance of the phase of superconducting wave-function.

\section{i) London Theory}

The discovery of the Meissner effect was soon ( 2 years later, in1935) followed by the work of $F$ London and $\mathrm{H}$ London, who proposed that flux density $B_{x}$ at a point ' $x$ ' within a superconductor is given as

$$
\frac{d^{2} B(x)}{d x^{2}}=\frac{B(x)}{\lambda_{L}^{2}}
$$

Where $\lambda_{\mathrm{L}}$ is the London penetration depth. The applied field $\mathrm{B}(\mathrm{x})$ penetrates into the superconductor within a very thin surface layer termed as penetration depth. If superconducting firm or filament is thinner than $\lambda_{L}$ its properties are significantly different from those of the bulk material. The solution of above differential equation in one dimension is

$$
B(x)=B(0) e^{-\cdot \frac{x}{\lambda_{L}}}
$$

Which shows that flux density decreases exponentially within the surface layer and essentially disappears when ' $\mathrm{x}$ ' is much greater than $\lambda_{\mathrm{L}}$, a result which accounts for the Meissner effect. The London's penetration depth is given by

$$
\lambda_{L}=\left(\frac{m c^{2}}{4 \pi n_{s} e^{2}}\right)^{\frac{1}{2}}, \lambda_{L} \text { is in the order of } \mu \text { m or } n m .
$$

\section{ii) Pippard Model on London's Theory}

The insufficiency in London's theory was modified by Pippard. He proposed a successful model, based on the uncertainty principle and energy distribution of the electrons. The model recognizes that only those electrons with energy within $\mathrm{KT}_{\mathrm{C}}$ of the Fermi energy will be involved in phenomena that occurs at $\mathrm{T}_{\mathrm{c}}$ and that those electrons have the Fermi velocity $\mathrm{V}_{\mathrm{F}}$. Thus from the uncertainty principal, the range of momentum $(\delta \mathrm{p})=\mathrm{KT}_{\mathrm{c}} / \mathrm{V}_{\mathrm{F}}$ and the characteristics length of the wave packet,

$\xi_{\mathrm{o}}=\frac{\mathrm{a} \hbar \mathrm{v}_{\mathrm{F}}}{2 \pi \mathrm{KT}_{\mathrm{c}}}$, 'a' being a constant.

\section{iii) Ginsburg - Landau Theory}

Ginsburg and Landau, proposed in 1950 a highly, innovative phenomenological theory of superconductivity based on Landau's theory of phase transition, which now bears their name. The theory introduced a temperature dependent coherence length $\xi(\mathrm{T})$. The Ginsburg-Landau parameters $\mathrm{K}_{\mathrm{GL}}=\lambda / \xi$ is a number, which determines the types of superconductors for type-I or type-II. If $\lambda>\xi$, it is a type- II superconductor and if $\lambda<$ $\xi$, it is a type-I superconductor. The properties of a superconductor depend sensitively on the G-L parameter, a dimensionless quantity. 
iv) Two Types of Superconductors

Type-I and type-II are the two kinds of superconductors. Type- I superconductors are characterized by perfect diamagnetism i.e. material completely expel magnetic flux until they become completely normal. The screening current flowing in the surface layer produces a magnetization which cancels the applied magnetic field in the interior of the superconductor. The strength of the applied magnetic field required to completely destroy the state of perfect diamagnetism in the interior of type-I superconducting material is called the thermodynamic critical field $\mathrm{H}_{\mathrm{C}}$, whose variation with temperature is governed by the relation

$$
H c=H c(0)\left[1-\left(\frac{T}{T_{C}}\right)^{2}\right]
$$

Where $\mathrm{Hc}(0)$ is the extrapolated Value of Ho at $\mathrm{T}=$ $0 \mathrm{~K}$. The value of Ho for mercury is 411 Gauss. This value is different for different material.

There are superconducting materials of typeII for which the magnetization varies with the applied field with two critical field designated as $\mathrm{Hc}_{1}$ and $\mathrm{Hc}_{2}$, the lower critical field and upper critical field respectively. The magnetic field is completely expelled only up to the field Hc. At Hc, the flux begins to penetrate the specimen and the penetration increases until $\mathrm{Hc}_{2}$ is reached. $\mathrm{At} \mathrm{Hc}_{2}$ the magnetization vanishes and the specimen becomes a normal conductor. Moreover, the magnetization of this group of superconductor vanishes gradually as the field is increased rather than suddenly as for type-I superconductors. On the other hand type-I Superconductor may be described by the non-localized modification of the London model and the type-II by the original localized London model.

\section{v) Cooper Pairs}

In the normal state of a metal, the conduction electrons behave like a gas of nearly free electrons that are scattered by lattice vibration, lattice imperfection etc, contribute to the resistivity. When we cool the specimen the normal state transforms itself into a different type of fluid, a quantum fluid of highly correlated pairs of electrons. Below $\mathrm{T}_{c}$ a conduction electron of a given momentum and spin get weakly coupled with another electron of exactly the opposite momentum and spin. These pairs of electrons are called cooper pairs. In total they have spin zero and have many attributes of bosons. The effective unit of charge is ' $2 \mathrm{e}$ ' rather than ' $\mathrm{e}$ ' as in ordinary state, where ' $\mathrm{e}$ ' is considered basic unit of charge. The glue is provided by the elastic waves of the lattice, called phonons .The distance between the two electrons between the Cooper pairs, called the coherence length, $\xi$. It has value of few angstroms (in pure $\mathrm{Nb} \sim 380 \mathrm{~A}^{\circ}$ ). The Cooper pairs are governed by certain requirements of quantum mechanics, the most important of which may be the requirement that all the Copper pairs have the same value of total momentum. A Cooper pair is more stable than two unpaired electrons by the amount of the binary energy. The Cooper pairs are broken up when energy is supplied to the metal, for example, by application of an external magnetic field or by increasing the temperature. The population of unpaired electrons is proportional to $\exp (-\mathrm{E} / \mathrm{KT})$. The attractive potential energy in BCS state reduces the total energy of the state than the Fermi state. As a consequence the BCS state becomes more stable than Fermi state and hence superconductivity persists.

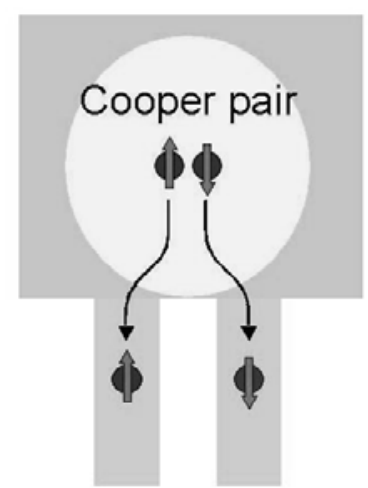

Fig2: When a Cooper pair of electrons moves from a superconducting material and sent into normally-conducting metal wires, the pair can be split up. A recent experiment studies how one electron can be sent down each of two wires, providing the wire spacing is less than the effective size of the Cooper Pair 
A simple picture of mechanism for superconductivity and the Cooper pairs can be summed up as follows. Consider one electron which is moving through the lattice of positive ions. The negative field of the electrons polarizes and distorts the lattice of positive ions to yield the region of excess positive ions which in turn, attract the second electron and yields an effective attractive interaction between the two electrons. If the attractive interaction is grater than the Coulomb repulsion, then phenomenon of superconductivity comes into play. The role of electron lattice interaction in the mechanism for superconductivity was first investigated by Fröhlich in 1950.

\section{vi) Bardeen, Cooper, Schrierieffer (BCS) Theory}

Out of various theories proposed on superconductivity, the most successful theory developed is by Bardeen, Cooper and Shrieffer (1957), known as BCS theory. According to the theory superconductivity occurs because of the existence an attractive force between conduction electrons, leading to bound pairs of electrons called Cooper pairs, which move without resistance.

The coulomb repulsive interaction in between two electrons is dominated by an attractive force arising due to electron- lattice-electron interaction. There is a negatively charged electron, which while moving through a lattice of positive ion attracts the positively charged ions. This distorts the lattice creating wave of excess positive change to which another electron of equal and opposite momentum and spin, is attracted to form a pair known as Cooper pair. These paired electrons are scattered like normal electrons, because charge in momentum in one of the electrons of a cooper paired electrons is counter-balanced by an equal and opposite change in momentum of the other electron. Hence, they can move through the material without résistance. A flow of such electron pairs continues a super-current. The theory has been extended and refined by numerous subsequent studies. All of the phenomena associated with the superconducting state such as specific heat, critical fields, tunneling, penetration depth $(\lambda)$, coherence length $(\xi)$, Meissner effect, and others have been successfully explained. The superconducting transition temperature of an element or alloy is given by

$$
K_{B} T_{C}=1.14 \hbar \omega_{D} e^{-\frac{1}{g N}(0)}
$$

Where ' $\mathrm{g}$ ' is the electron-phonon interaction, $\mathrm{N}(0)$ is the electron density of states of the Fermi level and $\theta_{\mathrm{D}}$ is the Debye temperature. For strong coupled superconductors, McMillan gives equation as,

$$
T_{C}=\frac{\theta_{D}}{1.45} e^{-\frac{1.04(1+\lambda)}{\lambda-\mu^{*}\left(1+<\omega>\frac{\lambda}{\omega_{0}}\right)}}
$$

Where $\langle\omega\rangle$ is the average phonon energy and $\mu^{*}$ is a coulomb pseudo potential. The parameter $\lambda$ is related to the electronic density of state through the equation

$$
\lambda=N(0) \frac{<I^{2}>}{M<\omega^{2}>}
$$

Where $<\mathrm{I}^{2}>$ is an electron phonon Matrix element averaged over the Fermi surface, $M$ is the atomic mass and $\left\langle\omega^{2}\right\rangle$ is the mean square phonon frequency. Equation (5) led to predict high temperature superconducting transitions.

\section{vii) Josephson Effect}

In 1962 B.D Josephson predicted theoretically that Cooper pairs tunneling through a very thin insulating layer $(2 \mathrm{~nm})$ is possible. Unpaired electrons tunneling takes place, if the insulting layer is relatively thicker. If the insulating barrier is made even thinner then Cooper pairs may tunnel from one side of the barrier to another side. There are important differences in the tunneling of unpaired electrons and the tunneling of Cooper pairs. The current with Cooper pair flows with zero resistance. Even in the absence of applied voltage, flow of electron takes place (dc Josephson effect ). On the other hand if a constant non-zero voltage were maintained across the tunnel barrier an alternating current (ac Josephson effect) would flow through the barrier in addition to the dc current produced by the tunneling of unpaired electrons. The magnitude of Josephson current is limited. If the current were above a sufficiently large value, the Cooper pairs passing through the barrier may have enough energy to dissociate into unpaired electrons. The unpaired electrons do not move with 
The Himalayan Physics, Vol.1, No.1, May 2010

zero resistance, nor do they move in the absence of a voltage difference. Josephson junctions are widely used in ultra sensitive magnetometers and voltmeters.

The de current across the junction of superconductor pairs is

$$
\mathrm{J}=\mathrm{J}_{0} \sin \delta=\mathrm{J}_{0} \sin \left(\theta_{2}-\theta_{1}\right)
$$

Where $\mathrm{J}_{0}$ is related to transfer interaction and is the maximum zero voltage current that can be passed by the junction and $\delta$ is the phase difference between the two wave functions. The current in ac Josephson effect is

$$
\mathrm{J}=\mathrm{J}_{0} \sin [\delta(0)-(2 \mathrm{eV} / \mathrm{h}) \mathrm{t}]
$$

Where $\mathrm{V}$ is applied dc voltage, $\hbar$ is a constant (Planck's constant h divided by $2 \pi$ ). The quantity in the parenthesis in above relation is the relative phase of the probability amplitude. The ac current oscillates with frequency, $\omega=2 \mathrm{eV} / \hbar$.

\section{Conclusion}

Finally, superconducting state is a distinct phase of matter having characteristic electrical, magnetic, and thermodynamic and other physical properties. The field of superconductivity has emerged as one of the most exciting fields of Solid State Physics. Soon after the discovery of superconductivity, a lot of research work have been undertaken to develop the theories and hence various superconducting behaviors of the superconducting materials like metals, alloys, semiconductors, organic compounds inter-metallic and interstitial compounds and so on. In the course of development plenty of ideas, theoretical as well as experimental achievements have been observed.

\section{References}

1. William E. Harfield and John H Miller, Jr. Marcel Dekker, (Editors) 1988. High Temperature Superconducting Materials, Preparation, properties and processing University of North Cardina Chapel Hill, North Canolina Marcel Dekker, Inc. New York and Basel.

2. S L Kakani and Shubhra kakani, 2007. Superconductivity, New Age International (P) Ltd, Publishers, New Delhi.

3. R Murugeshan and Kiruthiga Sivaprasath, 2007. Modern Physics, S. Chand and Company Ltd, Ram Nagar, New Delhi-110055

4. Neil W Ashcroftand N David Memin, Solid State Physics, 2007. Thomson Brooks/Cole, Australia.

5. Charles Kittel, 2004. Introduction to Solid State Physics, seventh Edition, Wiley India.

6. S L Kakani and C Hemarajani, 1991. Solid State Physic, Sultan Chand and Sons New Delhi110002.

7. R L Singhal, 1989. Solid state Physics, Kedr Nath Ram Nath \& Copany, Meerut.

8. R K Puri and V K Babbar, 1997. Solid State Physics, S Chand \& Company Ltd, New Delhi- 1 10055. 